\title{
A topological version of a theorem of Mather on twist maps
}

\author{
GLEN RICHARD HALL \\ Department of Mathematics, Boston University, Boston, MA 02215, USA
}

(Received 19 January 1984 and revised 21 May 1984)

\begin{abstract}
In this report we show that a twist map of an annulus with a periodic point of rotation number $p / q$ must have a Birkhoff periodic point of rotation number $p / q$. We use topological techniques so no assumption of area-preservation or circle intersection property is needed. If the map is area-preserving then this theorem and the fixed point theorem of Birkhoff imply a recent theorem of Aubry and Mather. We also show that periodic orbits of (significantly) smallest period for a twist map must be Birkhoff.
\end{abstract}

\section{Introduction}

We say a diffeomorphism $f$ of an annulus onto itself satisfies a twist condition if the angular component of the image of a point under $f$ increases as the radial component of the point increases (see $\S 2$ for precise definitions). Such maps were first studied in connection with the three-body problem by Poincaré, and in this context the map has a natural invariant measure. Birkhoff [4], [5] showed that such area-preserving twist maps have many periodic orbits, however his theorem gave no insight into the nature of these orbits. Recently, Aubry [2] and Mather [15] (see also [14]) have shown that area-preserving twist maps possess periodic orbits such that $f$ preserves the angular ordering of points on the orbit, such orbits are called Birkhoff periodic orbits (see [14] and $\S 2$ ). Moreover, they also showed the existence of 'quasi-periodic' orbits for area-preserving twist maps. The variational techniques used by Mather have proved useful in the study of other aspects of area-preserving twist maps (see, for example [16]).

Twist maps also occur frequently as non-area-preserving maps. For example, near the rest point of a map of the plane which has undergone Hopf bifurcation a twist condition will be satisfied. For such dissipative maps there will be no invariant measure.

In this report we show that a twist map of the annulus having a periodic orbit of some rotation number will have a Birkhoff periodic orbit of the same rotation number. One can think of this as replacing the area-preserving hypothesis with an assumption on the existence of periodic orbits, so this theorem can be applied to dissipative maps (see [9], [10]). Given an area-preserving twist map, Birkhoff's theorem mentioned above gives the existence of many periodic orbits, and hence the Aubry-Mather theorem may be derived as a corollary. 
The idea of the proof is to make use of the twist condition to show that periodic orbits which are not Birkhoff periodic orbits are topologically complicated. For example, if we suspend the twist map and look at the orbit of a non-Birkhoff periodic orbit it forms a non-trivial braid. The way in which these orbits link, given by the twist condition, can be used to imply the existence of new periodic points either by a geometric argument or a theorem on braids and periodic orbits of Matsuoka [17]. In particular, if a twist map has Birkhoff and non-Birkhoff periodic orbits of some given rotation number then it must in fact have two distinct Birkhoff periodic orbits with that rotation number.

In $\S 2$ we give the notation used throughout. In $\S 3$ we state the main result, proved in $\S 5$ using lemmas of $\S 4$. $\S 6$ is used to give some related theorems for twist maps which say basically that a periodic orbit with period (much) smaller than the period of every other periodic orbit of a given twist map must be a Birkhoff periodic orbit.

\section{Definitions and notation}

We let $A=\left\{(x, y) \in \mathbb{R}^{2}: 0 \leq y \leq 1\right\}, \pi_{1}$ (respectively $\left.\pi_{2}\right): A \rightarrow \mathbb{R}:(x, y) \rightarrow x$ (respectively $y)$, be the usual projections, and for each $\alpha \in \mathbb{R}$

$$
l_{\alpha}=\{(\alpha, y) \in A: 0 \leq y \leq 1\} .
$$

Definition. A map $f: A \rightarrow A$ is called a twist map if

(1) $f$ is a $C^{2}$ diffeomorphism, preserving boundary components and orientation;

(2) for all $(x, y) \in A, f((x, y))+(1,0)=f((x+1, y))$;

(3) there exists $\delta>0$, such that for all $z \in A, \partial\left(\pi_{1} \circ f\right)(z) / \partial y>\delta$.

Remarks. Condition (2) states that $f$ is the lift of a map on the annulus of which $A$ is the universal cover. Condition (3) is the 'twist condition', it implies that for any $\alpha, \beta \in \mathbb{R}, f\left(l_{\alpha}\right) \cap l_{\beta}$ is at most one point (see figure 1 ). This is sometimes called a 'monotone' twist condition.

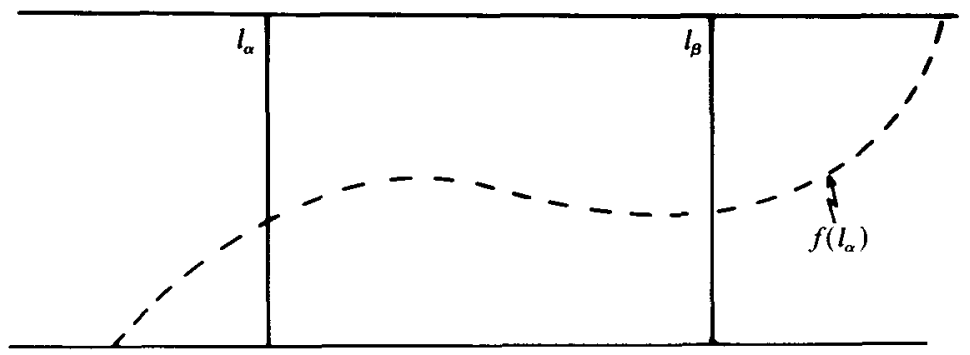

Figure 1

Definition. The orbit of a point $z \in A$ under a twist map $f: A \rightarrow A$ is defined to be the set $\mathcal{O}(f, z)=\left\{f^{k}(z)+(l, 0): k, l \in \mathbb{Z}\right\}$.

Definition. A point $z \in A$ is called a $p / q$-periodic point for a twist map $f: A \rightarrow A$ if

$$
f^{q}(z)-(p, 0)=z \text {. }
$$


Similarly, a point $z \in A$ is called a $p / q$-Birkhoff periodic point for $f$ if $z$ is a $p / q$-periodic point and for any $z_{1}, z_{2} \in \mathcal{O}(f, z)$

$$
\pi_{1}\left(z_{1}\right)<\pi_{1}\left(z_{2}\right) \Rightarrow \pi_{1}\left(f\left(z_{1}\right)\right)<\pi_{1}\left(f\left(z_{2}\right)\right) .
$$

Remarks. Recall that $f$ is the lift of an annulus map and hence the 'orbit' defined above and the definition of $p / q$-periodic point correspond to the lifts from the annulus of the usual orbit and periodic points. The $p / q$-Birkhoff periodic points are those $p / q$-periodic points $z$ for which $f$ restricted to $\mathscr{O}(f, z)$ is 'order preserving' in the $x$-coordinate.

Definition. If $f: A \rightarrow A$ is a twist map and $z \in A$ then the rotation number of $f$ at $z$ is

$$
\rho(f, z)=\lim _{n \rightarrow \infty} \frac{1}{n}\left(\pi_{1}\left(f^{n}(z)\right)\right)
$$

if it exists.

Notation. The maps $f_{\{(x, i): x \in \mathbb{R}\}}, i=0,1$ are lifts of circle diffeomorphisms, hence we let

$$
\rho_{0}(f)=\rho(f(x, 0)) \quad \text { and } \quad \rho_{1}(f)=\rho(f(x, 1))
$$

where these definitions are independent of $x \in \mathbb{R}$ and the limits exist (see [12]).

\section{Statement of the main theorem}

In this paper we prove the following theorem:

THEOREM 1. If $f: A \rightarrow A$ is a twist map and $f$ has a p/q-periodic point then $f$ has a $p / q$-Birkhoff periodic point.

This theorem applies whether or not $f$ is area-preserving. If $f$ is area-preserving then we can combine it with the following two results to obtain a theorem of Aubry and Mather.

THEOREM 2 (Birkhoff [4], [5], see also Chenciner [9]). Iff $: A \rightarrow A$ is an area-preserving twist map and $p / q \in\left[\rho_{0}(f), \rho_{1}(f)\right]$ then $f$ has a $p / q$-periodic point.

Remark. Birkhoff's theorem (known as 'Poincaré's Last Geometric Theorem') is actually true under a much weaker twist condition.

LEMMA 0 (Katok [14]). If $: A \rightarrow A$ is a twist map, $\left\{p_{n} / q_{n}\right\}_{n \geq 0}$ is a sequence of rationals with $p_{n} / q_{n} \rightarrow \alpha \notin \mathbb{Q}$ as $n \rightarrow \infty$ and for each $n, f$ has $a\left(p_{n} / q_{n}\right)$-Birkhoff periodic point $z_{n}$, then any limit point $z$ of $\left\{z_{n}\right\}_{n \geq 0}$ satisfies $\rho(f, z)=\alpha$.

Remark. In fact, much more is true of the orbit of such a limit point $z$ of $\left\{z_{n}\right\}_{n \geq 0}$. The map $f$ restricted to it is order preserving in the $x$-coordinate and the orbit lies on the graph of a periodic Lipschitz function (see [15], [14], [13]). These additional facts follow merely from the geometry of twist maps.

THEOREM 3 (Aubry [2] and Mather [15]). If $f: A \rightarrow A$ is an area-preserving twist map and $\alpha \in\left[\rho_{0}(f), \rho_{1}(f)\right]$ then there exists $z_{\alpha} \in A$ with $\rho\left(f, z_{\alpha}\right)=\alpha$. 
Proof of theorem 3. By Birkhoff's theorem (theorem 2), for each rational $p / q \in$ $\left[\rho_{0}(f), \rho_{1}(f)\right]$ there is a $z_{p / q} \in A$ which is a $p / q$-periodic point of $f$. By theorem 1 we see that there must then exist a $p / q$-Birkhoff periodic point. Applying lemma 0 to sequences of Birkhoff periodic points we can obtain points with irrational rotation number for any irrational in $\left[\rho_{0}(f), \rho_{1}(f)\right]$, which completes the proof of the theorem.

(See the remark above and the papers of Mather [15], Katok [14], and Herman [13] for the other properties of the orbits of these 'Birkhoff points'.)

The proof of theorem 1 proceeds as follows: Assume we have a twist map $f: A \rightarrow A$ with a $p / q$-periodic point. Then we show that $f$ is homotopic to a twist map $g: A \rightarrow A$ which has a $p / q$-Birkhoff periodic point such that each map in the homotopy has $p / q$-periodic points. The set of maps which have $p / q$-Birkhoff periodic points will then be seen to comprise an open-closed set in the parameter of this homotopy. Closure follows easily from the definition of Birkhoff periodic point (see lemma 1 below) while openness follows from the fixed point lemma of the next section, lemma 4. Basically this lemma says that a map with a $p / q$-Birkhoff periodic orbit and another $p / q$-periodic orbit must in fact have two $p / q$-Birkhoff periodic orbits, moreover this second orbit must persist under small perturbations.

The next section contains some lemmas needed for the proof of theorem 1 which is in $\S 5$. In $\S 6$ we show, using similar, but technically easier techniques that if $f: A \rightarrow A$ is a twist map and for some relatively prime integers $p, q$ the map $f$ satisfies the following condition:

( $\dagger$ ) Every $r / s$-periodic point of $f$ has $s=q$ or $s>\left(\frac{3}{2}\right) q$, then every $p / q$-periodic point of $f$ is a $p / q$-Birkhoff periodic point. Conditions on $p / q, \rho_{0}(f)$ and $\rho_{1}(f)$ can be given which imply the condition $(\dagger)$ above saying essentially that the map isn't twisting very much. For area-preserving twist maps we can improve this theorem, replacing $(\dagger)$ with the following:

( $\$$ ) Every $r / s$-periodic point of $f$ has $s \geq q$,

obtaining the same conclusion, that every $p / q$-periodic orbit is a $p / q$-Birkhoff periodic orbit.

Remark. A. Katok informed the author that D. Bernstein has recently given a proof of a version of theorem 3 for which the area-preservation property is replaced by the 'circle-intersection' property, see [3]. There is also a theorem of P. Carter [8] showing a result analogous to theorem 2 with area-preservation replaced with the circle-intersection property.

\section{Some lemmas}

In this section we prove lemmas useful in the proof of theorem 1.

LEMMA 1. Suppose $f_{n}: A \rightarrow A$ is a sequence of twist maps, $n=1,2, \ldots$, and for some fixed $p, q$ relatively prime integers, each $f_{n}$ has a $p / q$-Birkhoff periodic point $z_{n} \in A$. If $f_{n}$ converges, in the sup norm topology, to a twist map $f_{0}: A \rightarrow A$ (i.e. $\sup _{z \in A} \| f_{n}(z)-$ $f_{0}(z) \| \rightarrow 0$ as $n \rightarrow \infty$ where $\|\cdot\|$ is the usual $\mathbb{R}^{2}$ norm) and $z_{n}$ converges to $z_{0} \in A$ as $n$ tends to infinity then $z_{0}$ is a $p / q$-Birkhoff periodic point of $f_{0}$. 
Proof of lemma 1. Since $f_{n}^{q}\left(z_{n}\right)-(p, 0)=z_{n}$ for all $n=1,2, \ldots$, it follows that $f_{0}^{q}\left(z_{0}\right)-(p, 0)=z_{0}$. (Moreover, $f_{0}^{s}\left(z_{0}\right)-(r, 0) \neq z_{0}$ for any $r, s$ with $s<q$ since $p$ and $q$ are relatively prime.) Hence, $z_{0}$ is a $p / q$-periodic point of $f_{0}$.

To show that $z_{0}$ is a $p / q$-Birkhoff periodic point of $f_{0}$ we fix $k_{1}, k_{2}, l_{1}, l_{2} \in \mathbb{Z}$. Then for $n=1,2, \ldots$

$$
\pi_{1}\left(f_{n}^{k_{1}}\left(z_{n}\right)\right)+l_{1}<\pi_{1}\left(f_{n}^{k_{2}}\left(z_{n}\right)\right)+l_{2} \Rightarrow \pi_{1}\left(f_{n}^{k_{1}+1}\left(z_{n}\right)\right)+l_{1}<\pi_{1}\left(f_{n}^{k_{2}+1}\left(z_{n}\right)\right)+l_{2} .
$$

But then the same statement holds by continuity when we replace $f_{n}$ and $z_{n}$ by $f_{0}$ and $z_{0}$ respectively and put ' $\leq$ ' into the second inequality. But suppose

$$
\pi_{1}\left(f_{0}^{k_{1}}\left(z_{0}\right)\right)+l_{1}<\pi_{1}\left(f_{0}^{k_{2}}\left(z_{0}\right)\right)+l_{2}
$$

and

$$
\pi_{1}\left(f_{0}^{k_{1}+1}\left(z_{0}\right)\right)+l_{1}=\pi_{1}\left(f_{0}^{k_{2}+1}\left(z_{0}\right)\right)+l_{2} \text {. }
$$

Then, by the twist condition we have $\pi_{2}\left(f_{0}^{k_{1}+1}\left(z_{0}\right)\right)>\pi_{2}\left(f_{0}^{k_{2}+1}\left(z_{0}\right)\right)$ (see figure 2). So, again by the twist condition $\pi_{1}\left(f_{0}^{k_{1}+2}\left(z_{0}\right)\right)+l_{1}>\pi_{1}\left(f_{0}^{k_{2}+2}\left(z_{0}\right)\right)+l_{2}$. But this implies

$$
\pi_{1}\left(f_{n}^{k_{1}+2}\left(z_{n}\right)\right)+l_{1}>\pi_{1}\left(f_{n}^{k_{2}+2}\left(z_{n}\right)\right)+l_{2}
$$

for $n$ sufficiently large while $(*)$ above implies

$$
\pi_{1}\left(f_{n}^{k_{1}}\left(z_{n}\right)\right)+l_{1}<\pi_{1}\left(f_{0}^{k_{2}}\left(z_{n}\right)\right)+l_{2}
$$

for $n$ sufficiently large and this contradicts the fact that $z_{n}$ is a $p / q$-Birkhoff periodic point of $f_{n}$. So $z_{0}$ must be a $p / q$-Birkhoff periodic point of $f_{0}$ and the proof is complete.

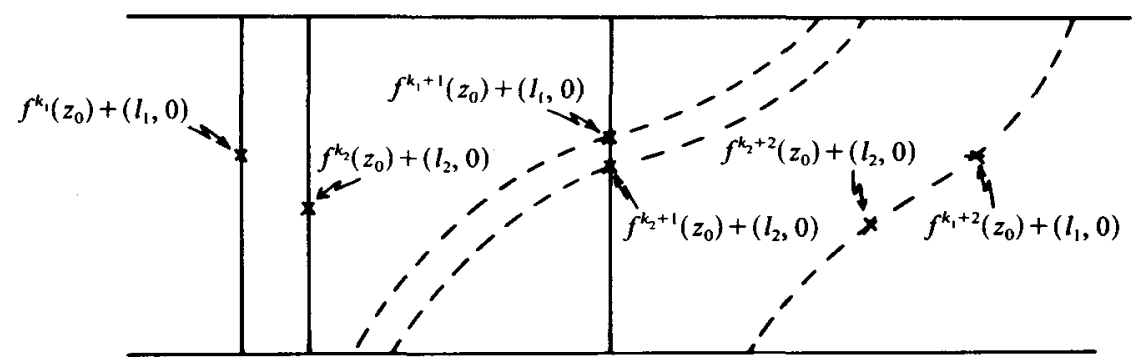

Figure 2

The next lemma says we can suspend a given twist map so that the intervening maps are also twist maps.

LEMMA 2. Given a twist map $f: A \rightarrow A$ there exists a $C^{1}$ map $\phi: A \times \mathbb{R} \rightarrow A$ satisfying

(1) $\phi(\cdot, 0)=$ identity on $A, \phi(\cdot, 1)=f(\cdot)$;

(2) for all $t \in[0,1]$ and all $n \in \mathbb{Z}, \phi(\cdot, t+n)=\phi\left(f^{n}(\cdot), t\right)$;

(3) for all $t \in[0,1], \phi(\cdot, t)$ is a twist map;

(4) for all $z \in A$ and all $t \in \mathbb{R}, \phi(z+(1,0), t)=\phi(z, t)+(1,0)$.

Proof of lemma 2. It suffices to define $\phi$ on $A \times[0,1]$ so that (1), (3) and (4) are satisfied since (2) can then be used to extend the definition to all of $\mathbb{R}$.

Let $f_{0}: \mathbb{R} \rightarrow \mathbb{R}$ be given by $f_{0}(x)=\pi_{1}(f(x, 0))$ and $G: \mathbb{R}^{2} \rightarrow \mathbb{R}^{2}$ be given by

$$
G(z)=D f\left(f^{-1}(z)\right)(0,1)
$$


for all $z \in A$, where $D f(w)$ is the derivative (matrix) of $f$ at $w \in A$. Then $f$ is determined by the map $f_{0}$ and the vector field $G$ as follows: Let $\psi$ be the local flow with domain in $A \times \mathbb{R}$ determined by the initial value problem

$$
\left\{\begin{array}{l}
\frac{d \psi}{d t}(z, t)=G(\psi(z, t)) \\
\psi(z, 0)=z, \quad \forall z \in A .
\end{array}\right.
$$

Then $f$ is given, for all $z \in A$, by

$$
f(z)=\psi\left(\left(f_{0}\left(\pi_{1}(z)\right), 0\right), \pi_{2}(z)\right) .
$$

The required homotopy of $f$ can then be easily constructed by:

(a) deforming $f_{0}$ to the identity on $\mathbb{R}$ through diffeomorphisms which are lifts of circle diffeomorphisms;

(b) forming a smooth, one-parameter family $G_{s}: A \rightarrow \mathbb{R}^{2}$ of vector fields with parameter $s \in[0,1]$ so that $G_{0}$ is the constant $(0,1)$ and $G_{1}$ is equal to $G$. To assure condition (3) we construct $G_{s}$ so that the angle between $G_{s}(z)$ and the $x$-axis is always between $-\pi / 2$ and $\pi / 2$ and increasing with $s$. For condition (4) we require $G_{s}(z+(1,0))=G_{s}(z)$ for all $z \in A, s \in[0,1]$.

The maps $\phi(\cdot, s)$ are now given by using the solution of the vector field $G_{s}$ and the initial conditions specified by the diffeomorphism in (a) above in an equation of the form (**). Of course, to extend $\phi$ to a globally smooth map on $A \times \mathbb{R}$ we must 'match up' the families of circle maps and vector fields given in (a) and (b) near $s=0$ and $s=1$. These details are left to the reader.

It is the topological nature of the orbits of $p / q$-Birkhoff periodic points under these flows which is the key to the proof of theorem 1, i.e. the fact that they are not 'linked', as is made precise in the next lemma.

Suppose $f: A \rightarrow A$ is a twist map and $p, q$ are relatively prime integers. Let $g: A \rightarrow A$ be defined for all $z \in A$ by

$$
g(z)=f^{q}(z)-(p, 0)
$$

Then the fixed points of $g$ correspond precisely with the $p / q$-periodic points of $f$. Suppose $f$ has a $p / q$-Birkhoff periodic point $z_{0} \in A$, then we have

Lemma 3. There exists a $C^{\prime}$ map $\phi: A \times \mathbb{R} \rightarrow A$ satisfying:

(i) for all $z \in A$ and all $t \in \mathbb{R}, \quad \phi(z+(1,0), t)=\phi(z, t)+(1,0)$;

(ii) $\phi(\cdot, 0)=$ identity, $\phi(\cdot, 1)=g(\cdot)$;

(iii) for all $t \in[0,1]$ and all $n \in \mathbb{Z}, \quad \phi(\cdot, t+n)=\phi\left(g^{n}(\cdot), t\right)$;

(iv) for all $t \in[0,1 / q]$ and $i=0, \ldots, q-1, \quad \phi\left(\phi(\cdot, i / q)^{-1}, i / q+t\right)$ is a twist map;

(v) for $i=1, \ldots, q$, and any $z, w \in A, \quad \pi_{1}(\phi(z, i / q))<\pi_{1}(\phi(w, i / q))$ if and only if $\pi_{1}\left(f^{i}(z)\right)<\pi_{1}\left(f^{i}(w)\right)$;

(vi) for all $\zeta \in \mathcal{O}\left(f, z_{0}\right)$ and all $t \in \mathbb{R}, \quad \phi(\zeta, t)=\zeta$.

Proof of lemma 3. Let $\phi_{1}: A \times \mathbb{R} \rightarrow A$ be the one parameter family associated with $f$ by lemma 2 . We may assume, by composing the maps $\phi_{1}(\cdot, t)$ with a map which preserves the $x=$ constant foliation (i.e. adjusting the norms of the vectors given by 
the vector field $\left.G_{t}\right)$, that if $\zeta_{1}, \zeta_{2} \in \mathscr{O}\left(f, z_{0}\right)$ with $\pi_{1}\left(\zeta_{1}\right)<\pi_{1}\left(\zeta_{2}\right)$ then

$$
\pi_{1}\left(\phi_{1}\left(\zeta_{1}, t\right)\right)<\pi_{1}\left(\phi_{1}\left(\zeta_{2}, t\right)\right) \quad \text { for all } t \in[0,1] \text {. }
$$

But then letting $\phi_{2}: A \times[0,1] \rightarrow A$ be defined for all $z \in A$ and all $t \in[0,1]$ by

$$
\phi_{2}(z, t)=\phi_{1}(z, q t)-t(p, 0)
$$

we see $\phi_{2}$ satisfies conditions (i), (ii), (iv) and (v) and that if $\zeta_{1}, \zeta_{2} \in \mathscr{O}\left(f, z_{0}\right)$, $\pi_{1}\left(\zeta_{1}\right)<\pi_{1}\left(\zeta_{2}\right)$ then $\pi_{1}\left(\phi_{2}\left(\zeta_{1}, t\right)\right)<\pi_{1}\left(\phi_{2}\left(\zeta_{2}, t\right)\right)$ for all $t \in[0,1]$. Hence, we may deform $\phi_{2}$ to a family $\phi: A \times[0,1] \rightarrow A$ so that (i), (ii), (iv), (v) and (vi) are satisfied and extend $\phi$ to a map on $\mathbb{R}$ by condition (iii). This is the required map $\phi$ and the proof is complete.

Remarks. (1) Informally we can say that the braid given by $\phi_{2}(\zeta, t)$ for $\zeta \in \mathscr{O}\left(f, z_{0}\right)$ is trivial hence it may be 'straightened out', (see figure 3 ).
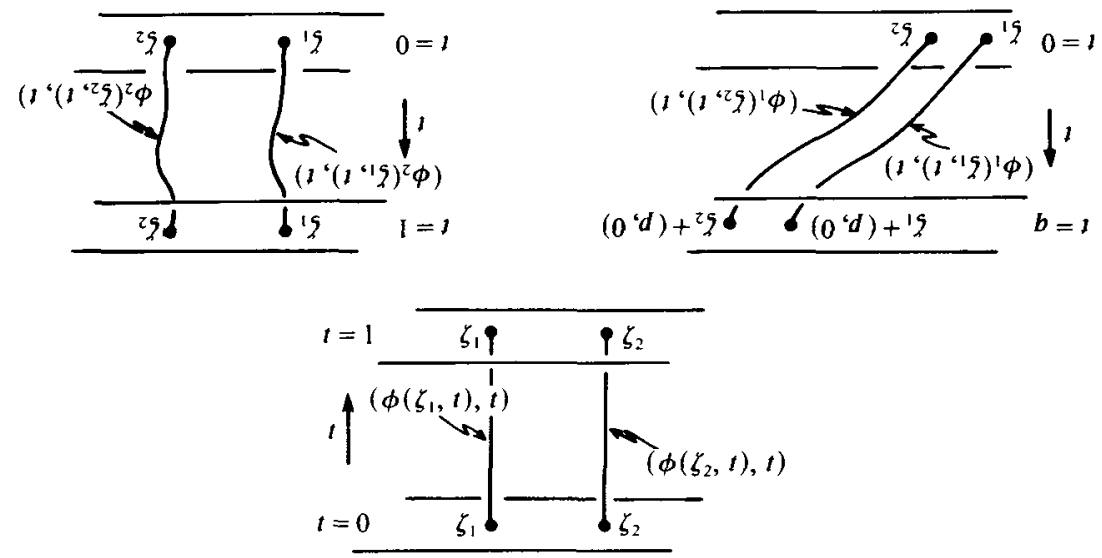

FIGURE 3

(2) The hypothesis that $z_{0}$ be a $p / q$-Birkhoff periodic point is necessary in the above lemma. In fact, if $z_{0}$ is a $p / q$-periodic point, but not a $p / q$-Birkhoff periodic point then the above lemma can not hold. To see this, note that if $z_{0}$ is not a $p / q$-Birkhoff periodic point then there exist $\zeta_{1}, \zeta_{2} \in \mathcal{O}\left(f, z_{0}\right)$ with $\pi_{1}\left(\zeta_{1}\right)<\pi_{1}\left(\zeta_{2}\right)$ and $\pi_{1}\left(f\left(\zeta_{1}\right)\right)>\pi_{1}\left(f\left(\zeta_{2}\right)\right)$. But as we will see in the proof of the next lemma, this implies that the orbits of $\zeta_{1}$ and $\zeta_{2}$ under $\phi_{1}$ 'link' non-trivially (see figure 4) and hence cannot be straightened out simultaneously.

(3) Finally we note that the proof of lemma 3 relies only on the fact that points of a Birkhoff periodic orbit stay in order, i.e. the same proof serves to show

Lemma $3^{\prime}$. With $f: A \rightarrow A, g: A \rightarrow A$ as above and $\zeta_{1}, \zeta_{2}$ p/q-periodic points of $f$ satisfying $\pi_{1}\left(f^{i}\left(\zeta_{1}\right)\right)<\pi_{1}\left(f^{i}\left(\zeta_{2}\right)\right)$ for all $i=0, \ldots, q$ there exists a map $\phi: A \times \mathbb{R} \rightarrow A$ satisfying (ii)-(v) of lemma 3 and

(vi') for all $t \in \mathbb{R}, \quad \phi\left(\zeta_{i}, t\right)=\zeta_{i}, i=1,2$.

Remark. It is important to note that we cannot require condition (i), periodicity in $x$, for this lemma since $\zeta_{1}$ may not link with $\zeta_{2}$ while linking with $\zeta_{2}-(1,0)$. Hence 


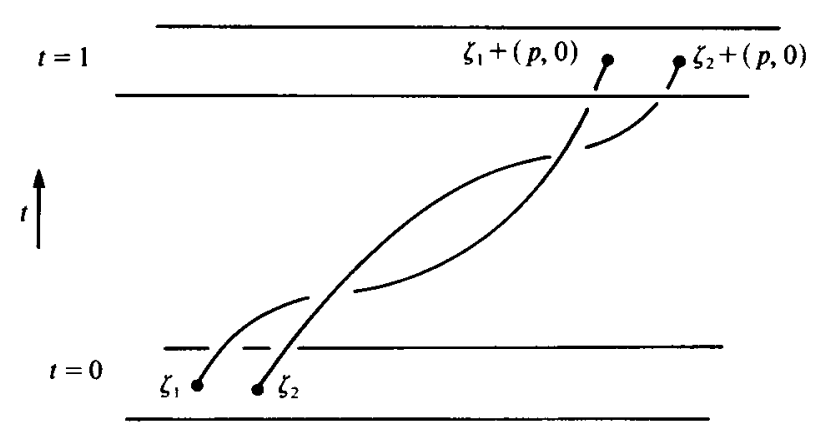

FIGURE 4

the 'straightening out' in this case takes place only in the covering space $A$, not in the annulus.

Proof of lemma 3'. Same as the proof of lemma 3.

The final lemma of this section is the 'fixed point' lemma required for theorem 1.

LeMMA 4. Suppose $f: A \rightarrow A$ is a twist map and $p, q$ are relatively prime integers. Suppose $f$ has a $p / q$-Birkhoff periodic point $z_{0} \in A$ and $a p / q$-periodic point $w_{0} \in A$ which is not a Birkhoff periodic point. Then $f$ has another $p / q$-Birkhoff periodic point $z_{1} \in A$ with $z_{1} \notin \mathcal{O}\left(f, z_{0}\right)$. Moreover, if $\tilde{f}: A \rightarrow A$ is a twist map and $\sup _{z \in A}\|f(z)-\tilde{f}(z)\|$ is sufficiently small, where $\|\cdot\|$ is the usual norm in $\mathbb{R}^{2}$, then $\tilde{f}$ has a $p / q$-Birkhoff periodic point.

Remark. This lemma is reminiscent of the theorem of Birkhoff for area-preserving twist maps (of which theorem 2 in $\S 3$ is a corollary) which states that if $0 \epsilon$ $\left[\rho_{0}(f), \rho_{1}(f)\right]$ for a twist map $f$ then $f$ must have two fixed points. That $f$ must have two fixed points in the generic case follows easily from index arguments, however $f$ must have two fixed points even when they are degenerate (see [4], [5] and [7]). It should be emphasized that we are not assuming area-preservation or any circle intersection properties in lemma 4.

Proof of lemma 4. Let $g(z)=f^{q}(z)-(p, 0)$ for all $z \in A$ as above and let $\phi: A \times \mathbb{P} \rightarrow A$ be the one parameter family associated with $g$ and the orbit of $z_{0} \in A$ by lemma 3 . For convenience we introduce the following technical notation:

We say two points $\zeta, \eta \in A$ get out of order if $\pi_{1}(\zeta)<\pi_{1}(\eta)$ and $\pi_{1}\left(f^{i}(\zeta)\right)>$ $\pi_{1}\left(f^{i}(\eta)\right)$ for some $i, 0<i<q$, or if $\pi_{1}(\zeta)>\pi_{1}(\eta)$ and $\pi_{1}\left(f^{i}(\zeta)\right)<\pi_{1}\left(f^{i}(\eta)\right)$ for some $i, 0<i<q$.

An immediate consequence is that if $\zeta, \eta$ are $p / q$-periodic points of $f$ then $\zeta, \eta$ get out of order if and only if $f^{k}(\zeta), f^{k}(\eta)$ get out of order for all $k \in \mathbb{Z}$.

Let $\mu_{0}, \mu_{1}, \ldots, \mu_{q-1} \in \mathcal{O}\left(f, z_{0}\right)$ be chosen and ordered so that

$$
0 \leq \pi_{1}\left(\mu_{0}\right)<\pi_{1}\left(\mu_{1}\right)<\cdots<\pi_{1}\left(\mu_{q-1}\right)<1
$$

and let $\nu_{0}, \ldots, \nu_{q-1} \in \mathcal{O}\left(f, w_{0}\right)$ be chosen and ordered so that

$$
\pi_{1}\left(\mu_{0}\right) \leq \pi_{1}\left(\nu_{0}\right)<\pi_{1}\left(\nu_{1}\right)<\cdots<\pi_{1}\left(\nu_{q-1}\right)<\pi_{1}\left(\mu_{0}+(1,0)\right) \text {. }
$$

(We may assume $\pi_{1}\left(\nu_{i}\right) \neq \pi\left(\nu_{i+1}\right)$ by changing coordinates slightly if necessary.) 
Now we specify two cases by the Pigeon Hole Principle:

Case 1. There exists $i_{0}, 0 \leq i_{0}<q$, such that for all $j=0,1, \ldots, q-1, \pi_{1}\left(\nu_{j}\right) \notin\left[\pi_{1}\left(\mu_{i_{0}}\right)\right.$, $\pi_{1}\left(\mu_{i_{0}+1}\right)$ ) (or $\pi_{1}\left(\nu_{i}\right) \notin\left[\pi_{1}\left(\mu_{q-1}\right)_{2} \pi_{1}\left(\mu_{0}\right)+1\right)$ if $\left.i_{0}=q-1\right)$.

Case 2. For each $i, 0 \leq i<q, \pi_{1}\left(\nu_{i}\right) \in\left[\pi_{1}\left(\mu_{i}\right), \pi_{1}\left(\mu_{i+1}\right)\right)$ (and $\pi_{1}\left(\nu_{q-1}\right) \in\left[\pi_{1}\left(\mu_{q-1}\right)\right.$, $\left.\pi_{1}\left(\mu_{0}\right)+1\right)$.)

If case 1 holds take $\zeta_{0}=\mu_{i_{0}}$ and $\zeta_{1}=\mu_{i_{0}+1}$; in case 2 take $\zeta_{0}=\mu_{0}$ and $\zeta_{1}=\mu_{1}$.

Next we note that since $w_{0}$ is not a Birkhoff periodic point, there must exist $z \in \mathcal{O}\left(f, z_{0}\right)$ such that $z, w_{0}$ get out of order. Let $\bar{z} \in \mathscr{O}\left(f, z_{0}\right)$ be such that $\bar{z}, w_{0}$ get out of order and if $z \in \mathscr{O}\left(f, z_{0}\right)$ and $z, w_{0}$ get out order then $z=\bar{z}$ or $\pi_{1}(z)<\pi_{1}(\bar{z})$. Similarly let $z \in \mathcal{O}\left(f, z_{0}\right)$ be such that $z, w_{0}$ get out of order and if $z \in \mathcal{O}\left(f, w_{0}\right)$ and $z, w_{0}$ get out of order then $\underline{z}=z$ or $\pi_{1}(z)<\pi_{1}(z)$, (it is possible that $\bar{z}=\underline{z}$ ). Fix $r_{1}, r_{2}, s_{1}, s_{2} \in \mathbb{Z}$ so that $f^{r_{1}}(\bar{z})=\zeta_{0}+\left(s_{1}, 0\right)$ and $f^{r_{2}}(\underline{z})=\zeta_{1}+\left(s_{2}, 0\right)$. Then it follows that $\zeta_{0}+\left(s_{1}, 0\right), f^{r_{1}}\left(w_{0}\right)$ get out of order and $\zeta_{1}+\left(s_{2}, 0\right), f^{r_{2}}\left(w_{0}\right)$ get out of order. Letting

$$
\eta_{0}=f^{r_{1}}\left(w_{0}\right)-\left(s_{1}, 0\right), \quad \eta_{1}=f^{r_{2}}\left(w_{0}\right)-\left(s_{2}, 0\right)
$$

we have that $\zeta_{0}, \eta_{0}$ get out of order and $\zeta_{1}, \eta_{1}$ get out of order. Now, $\zeta_{0}, \eta_{1}$ do not get out of order, since if they did then $\zeta_{0}+\left(s_{2}, 0\right), f^{r_{2}}\left(w_{0}\right)$ would get out of order, so $f^{-r_{2}}\left(\zeta_{0}+\left(s_{2}, 0\right)\right)$, $w_{0}$ would get out of order. But $z_{0}$ is a Birkhoff periodic orbit, so $\pi_{1}\left(\zeta_{0}\right)<\pi_{1}\left(\zeta_{1}\right)$ implies

$$
\pi_{1}\left(f^{-r_{2}}\left(\zeta_{0}+\left(s_{2}, 0\right)\right)\right)<\pi_{1}\left(f^{-r_{2}}\left(\zeta_{1}+\left(s_{2}, 0\right)\right)=\pi_{1}(\underline{z})\right.
$$

and we have a contradiction of the choice of $z$. Similarly, $\zeta_{1}, \eta_{0}$ do not get out of order, i.e. for $i=0, \ldots, q-1, \pi_{1}\left(f^{i}\left(\zeta_{0}\right)\right)<\pi_{1}\left(f^{i}\left(\eta_{1}\right)\right)$ and $\pi_{1}\left(f^{i}\left(\zeta_{1}\right)\right)>\pi_{1}\left(f^{i}\left(\eta_{0}\right)\right)$. If we are in case 2 then either

(i) $\pi_{1}\left(\eta_{0}\right)<\pi_{1}\left(\zeta_{0}\right)$ and $\pi_{1}\left(\zeta_{1}\right) \leq \pi_{1}\left(\eta_{1}\right)$;

(ii) $\pi_{1}\left(\eta_{0}\right)<\pi_{1}\left(\zeta_{0}\right)$ and $\pi_{1}\left(\zeta_{0}\right) \leq \pi_{1}\left(\eta_{1}\right)<\pi_{1}\left(\zeta_{1}\right)$; or

(iii) $\pi_{1}\left(\zeta_{0}\right) \leq \pi_{1}\left(\eta_{0}\right)<\pi_{1}\left(\zeta_{1}\right)$ and $\pi_{1}\left(\zeta_{1}\right)<\pi_{1}\left(\eta_{1}\right)$;

while in case 1 condition (i) must hold.

Suppose $\pi_{1}\left(\eta_{0}\right)<\pi_{1}\left(\zeta_{0}\right)$ and for some $i, 0<i<q, \pi_{1}\left(f^{i}\left(\eta_{0}\right)\right)>\pi_{1}\left(f^{i}\left(\zeta_{0}\right)\right)$. Fix the smallest $i>0$ such that $\pi_{1}\left(\phi\left(\eta_{0},(i-1) / q\right)\right)<\pi_{1}\left(\zeta_{0}\right)$ and $\pi_{1}\left(\phi\left(\eta_{0}, i / q\right)\right)>\pi_{1}\left(\zeta_{0}\right)$. By the twist condition we see that if $t \in[0,1 / q)$ and $\pi_{1}\left(\phi\left(\eta_{0}, i / q+t\right)\right)=\pi_{1}\left(\zeta_{0}\right)$ then

$$
\pi_{2}\left(\phi\left(\eta_{0},(i-1) / q+t\right)\right)>\pi_{2}\left(\zeta_{0}\right) .
$$

Fixing the smallest $j>i$ such that $\left.\pi_{1}\left(\phi\left(\eta_{0},(j-1)\right) / q\right)\right)>\pi_{1}\left(\zeta_{0}\right)$ and $\pi_{1}\left(\phi\left(\eta_{0}, j / q\right)\right) \leq \pi_{1}\left(\zeta_{0}\right)$ we see similarly that if $t \in[0,1 / q)$ and $\pi_{1}\left(\phi\left(\eta_{0},(j-1) / q+t\right)\right.$ $=\pi_{1}\left(\zeta_{0}\right)$ then

$$
\pi_{2}\left(\phi\left(\eta_{0},(j-1) / q+t\right)\right)<\pi_{2}\left(\zeta_{0}\right) .
$$

Repeating this argument until $i>q$ we see that $\phi\left(\eta_{0},[0,1]\right)$ is not contractible in $A \sim\left\{\zeta_{0}\right\}$. Similarly, this statement holds if $\pi_{1}\left(\eta_{0}\right) \geq \pi_{1}\left(\zeta_{0}\right)$. Also, the same argument implies that $\phi\left(\eta_{1},[0,1]\right)$ is not contractible in $A \sim\left\{\zeta_{1}\right\}$. Schematically the situation is as pictured in figure 5 .

Claim. There exists a point $z_{1} \in A, \pi_{1}\left(\zeta_{0}\right)<\pi_{1}\left(z_{1}\right)<\pi_{1}\left(\zeta_{1}\right)$, such that $z_{1}$ is a fixed point of $g$ and the loop $\phi\left(z_{1},[0,1]\right)$ is contractible in $A \sim\left\{\zeta_{0}, \zeta_{1}\right\}$. 

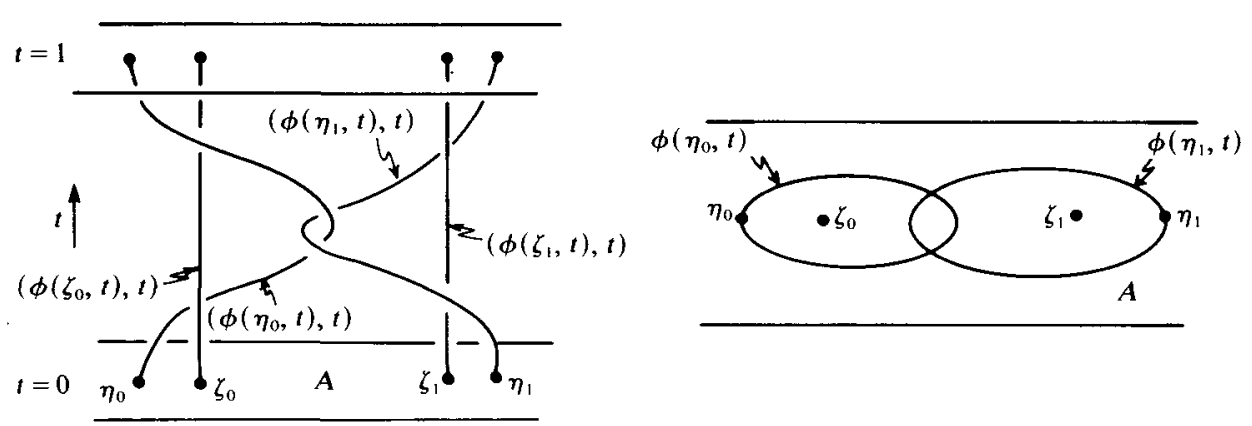

Figure 5

Remarks. (1) There are several alternatives for the proof of this claim. As indicated in figure 5 , the orbits of $\zeta_{0}, \zeta_{1}, \eta_{0}$ and $\eta_{1}$ form a braid of a fairly simple form and hence it is not surprising that a proof of the claim can be obtained via a computation and an application of a theorem of Matsuoka [17] on braids and periodic orbits for time periodic o.d.e.'s on surfaces. The elementary proof given below gives a geometrical view of some simple cases of Matsuoka's theorem. (See also recent work of Asimov and Franks [1] relating 'removable' periodic orbits and Nielson theory.)

(2) The author would like to thank M. Handel for suggestions regarding this proof, particularly that the 'stability' statement of lemma 4 could be shown via the geometrical approach. This simplifies several steps in the next section.

Proof of the claim. First we show the proof for case 1 ; case 2 follows from similar, easier, arguments.

Case 1: $\left(\pi_{1}\left(\eta_{0}\right)<\pi_{1}\left(\zeta_{0}\right)\right.$ and $\left.\pi_{1}\left(\eta_{1}\right) \geq \pi_{1}\left(\zeta_{1}\right)\right)$. The following notation will be useful. Let

$$
\begin{aligned}
B & =\left\{z \in A: \pi_{1}\left(\zeta_{0}\right) \leq \pi_{1}(z) \leq \pi_{1}\left(\zeta_{1}\right)\right\} & & \\
b_{i}^{+} & =\left\{\left(\pi_{1}\left(\zeta_{i}\right), y\right) \in A: y>\pi_{2}\left(\zeta_{i}\right)\right\}, & & i=0,1 \\
b_{i}^{-} & =\left\{\left(\pi_{1}\left(\zeta_{i}\right), y\right) \in A: y<\pi_{2}\left(\zeta_{i}\right)\right\}, & & i=0,1 \quad \text { (see figure 6) }
\end{aligned}
$$

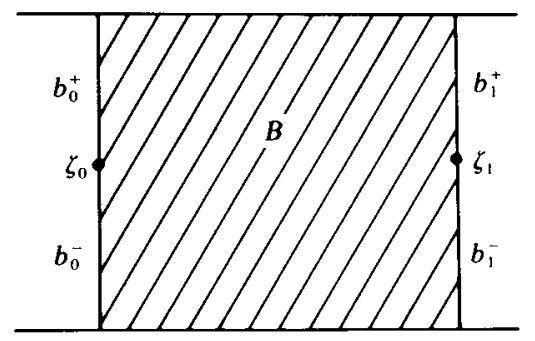

FIGURE 6

The following definition will also be useful:

For $t \in[0,1]$ we say a point $\zeta \in B$ with $\phi(\zeta, t) \in \phi(B, t) \cap B$ is null in $B$ at time $t$ if the loop formed by $\phi(\zeta,[0, t])$ and the segment joining $\phi(\zeta, t)$ and $\zeta$ is contractible 
in $A \sim\left\{\zeta_{0}, \zeta_{1}\right\}$. We let

$S_{t}=\{\phi(\zeta, t) \in B: \zeta \in B$ and is null in $B$ at time $t\}$.

Remark. Several other characterizations of $S_{t}$ are available. For example, if we let $\hat{A}$ be the universal covering space of $A \sim\left\{\zeta_{0}, \zeta_{1}\right\}, \hat{\phi}: \hat{A} \times \mathbb{R} \rightarrow \hat{A}$ the lift of $\phi$ with $\hat{\phi}(\cdot, 0)$ the identity on $\hat{A}$ and $\hat{B} \subseteq \hat{A}$ a particular fixed lift of $B$ then the points $\zeta \in S_{t}$ are precisely the projections of the points in $\hat{\phi}(\hat{B}, t) \cap \hat{B}$.

We note that if $\zeta$ is null in $B$ then it does not necessarily follow that $\phi(\zeta,[0, t]) \subseteq B$. However, it does follow that $\phi(\zeta, i / q) \in B$ whenever $0 \leq i / q \leq t$ and that if $\pi_{1}\left(\phi\left(\zeta, t^{\prime}\right)\right)=\pi_{1}\left(\zeta_{i}\right), i=0$ or 1 for some $t^{\prime} \in[0, t]$ then $\phi\left(\zeta, t^{\prime}\right) \in b_{0}^{-} \cup b_{1}^{+}$. This follows from the twist condition.

Similarly, the twist condition implies that for each $t \in[0,1]$, we have

$$
S_{t} \cap\left(b_{0}^{+} \cup b_{1}^{-}\right)=\varnothing
$$

and if $\sigma:[0,1] \rightarrow B$ has $\sigma(0) \in b_{0}^{-}, \sigma(1) \in b_{1}^{+}$then for each $t \in[0,1], \phi(\sigma([0,1]), t) \cap S_{t}$ contains an arc with one end point in $b_{0}^{-}$and the other in $b_{1}^{+}$. Finally, we note that $\partial S_{t} \cap$ (interior $B$ ) is made up of arcs of the form $\phi(J, t)$ where $J$ is an interval in $b_{0}^{+}$or $b_{1}^{-}$and

$$
\partial S_{t} \cap \partial B \subseteq b_{0}^{-} \cup b_{1}^{+} \cup\left\{\zeta_{0}, \zeta_{1}\right\} \cup\{(x, j) \in B: j=0,1\} .
$$

Next we use the existence of $\eta_{0}$ and $\eta_{1}$ and the properties of their orbits under $\phi$ to show that when $t=1, S$, must contain a connected component $T_{t}$ such that

( $\alpha) \partial T_{t} \cap\left\{\zeta_{0}, \zeta_{1}\right\}=\varnothing$;

( $\beta$ ) $\partial T_{t}$ contains arcs of the form $\phi\left(J_{0}, t\right), \phi\left(J_{1}, t\right)$ where $J_{0} \subseteq b_{0}^{+}, J_{1} \subseteq b_{1}^{-}$and both $\phi\left(J_{0}, t\right)$ and $\phi\left(J_{1}, t\right)$ contain points of both $b_{0}^{-} \cup\{(x, 0) \in B\}$ and $b_{1}^{+} \cup$ $\{(x, 1) \in B\}$, (see figure 7 ).

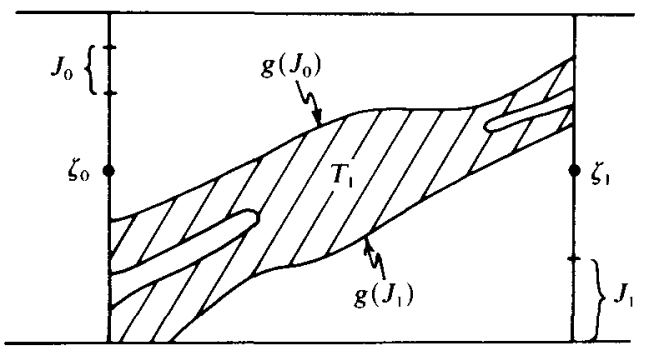

FIGURE 7

An easy index argument applied to the set $g^{-1}\left(T_{1}\right)$ will then give the required fixed point $z_{1} \in A$.

Fix the smallest $i_{0}, i_{1}$ such that $\phi\left(\eta_{0}, i_{0} / q\right), \phi\left(\eta_{1}, i_{1} / q\right) \in B$ and the smallest $j_{0}>i_{0}$, $j_{1}>i_{1}$ such that $\phi\left(\eta_{0}, j_{0} / q\right), \phi\left(\eta_{1}, j_{1} / q\right) \notin B$. We assume first that $j_{0} \leq j_{1}$ and consider the following cases:

Case a. For all $i=i_{0}, i_{0}+1, \ldots, j_{0}, \quad \pi_{1}\left(\phi\left(\eta_{0}, i / q\right)\right)<\pi_{1}\left(\phi\left(\eta_{1}, i / q\right)\right)$.

Case b. For some $i, i_{0} \leq i<j_{0}, \quad \pi_{1}\left(\phi\left(\eta_{0}, i / q\right)\right)>\pi_{1}\left(\phi\left(\eta_{1}, i / q\right)\right)$, (i.e. either the orbits of $\eta_{0}, \eta_{1}$ do not link, or they do, respectively). 
Case a. The loop formed by $\phi\left(\eta_{0},\left[0, j_{0} / q\right]\right)$ followed by the segment connecting $\phi\left(\eta_{0}, j_{0} / q\right)$ and $\eta_{0}$ is not contractible in $A \sim\left\{\zeta_{0}\right\}$. Hence, $S_{j_{0} / q}$ must contain a component $T_{j_{0} / q}$ satisfying $(\beta)$ above and $\zeta_{0} \notin T_{j_{0} / q}$ (see figure $8 \mathrm{~b}$ ).

Similarly, $\phi\left(\phi^{-1}\left(T_{j_{0} / q}, j_{0} / q\right), j_{1} / q\right)$ must contain a component $T_{j_{1} / q}$ satisfying $(\alpha)$ and $(\beta)$ and $g\left(\phi^{-1}\left(T_{j_{1} / q}, j_{1} / q\right)\right)$ will therefore contain the desired component of $S_{1}$ (see figure $8 \mathrm{c}$ ).

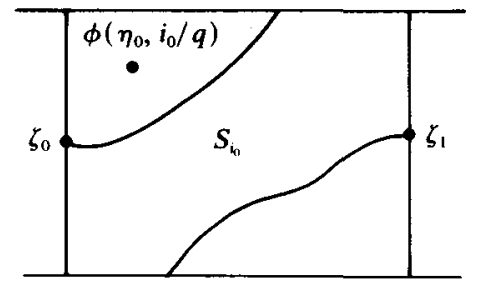

(a)

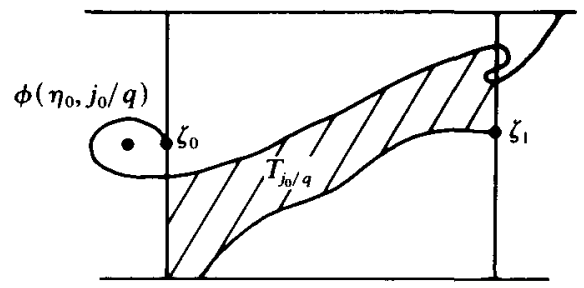

(b)

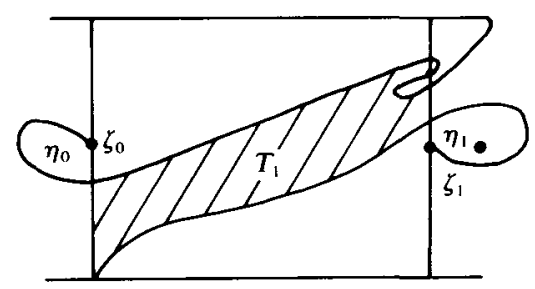

(c)

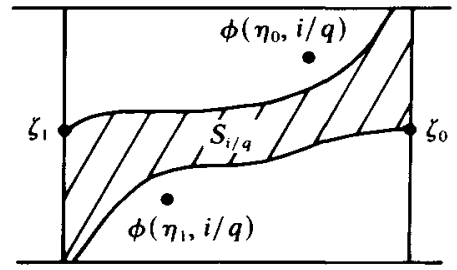

(d)

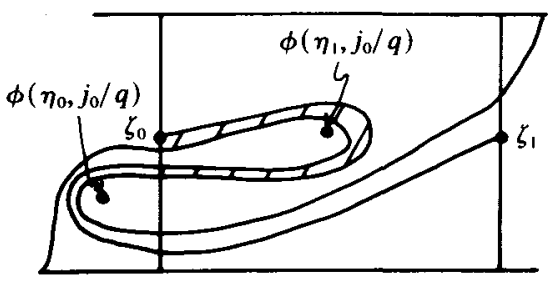

(f)

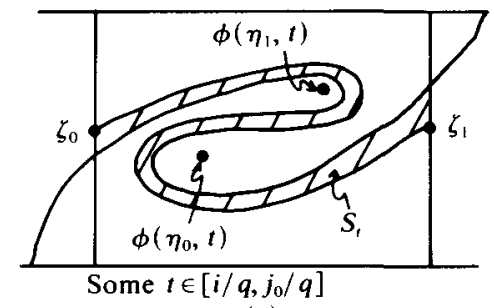

(e)

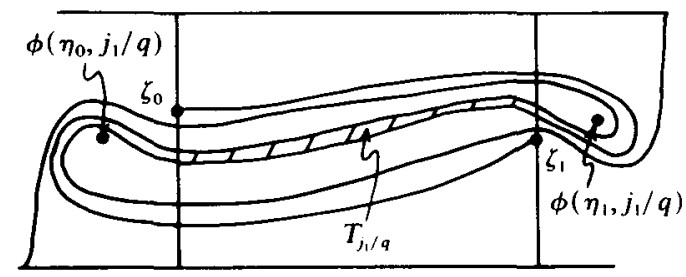

(g)

FIGURE 8

In fact, in this case we also have that

$$
\pi_{1}\left(\phi\left(\eta_{0}, i / q\right)\right)<\pi_{1}(\phi(z, i / q))<\pi_{1}\left(\phi\left(\eta_{1}, i / q\right)\right)
$$

for $z \in g^{-1}(T), i=0, \ldots, q$, so no point in $g^{-1}(T)$ gets out of order with $\eta_{0}$ or $\eta_{1}$. (This will be used in case 2 below.) 
Case b. Since the loop $\phi\left(\eta_{0},\left[0, j_{0} / q\right]\right)$ followed by the segment connecting $\eta_{0}$ to $\phi\left(\eta_{0}, j_{0} / q\right)$ is not contractible in $A \sim\left\{\zeta_{0}\right\}$, and $\pi_{1}\left(\phi\left(\eta_{0}, i / q\right)\right)>\pi_{1}\left(\phi\left(\eta_{1}, i / q\right)\right)$ for some $i, 0<i<j_{0}$, we see that $S_{j_{0} / q}$ either contains a component $T_{j_{0} / q}$ satisfying $(\alpha)$ and $(\beta)$ above (in which case a component of $g\left(\phi^{-1}\left(T_{j_{0} / q}, j_{0} / q\right)\right)$ is the required set) or $S_{j_{0} / q}$ contains a component $T_{j_{0} / q}$ disjoint from $\left\{\zeta_{1}\right\}$ with $\partial T_{j_{0} / q}$ containing arcs $\phi\left(J_{0}, j_{0} / q\right)$ and $\phi\left(J_{1}, j_{0} / q\right)$ where $J_{0}, J_{1}$ are intervals in $b_{0}^{+}, b_{1}^{-}$respectively, and $\phi\left(J_{0}, j_{0} / q\right)$ has both end points in $b_{0}^{-}$while $\phi\left(J_{1}, j_{0} / q\right)$ either has both end points in $b_{0}^{-}$or one end point in $b_{0}^{-} \sim\left\{\zeta_{0}\right\}$ and one in $b_{1}^{+} \sim\left\{\zeta_{1}\right\}$ (see figure 8f). When both end points of these arcs are in $b_{0}^{-}$the arcs are not homotopic to $b_{0}^{-}$in $B \sim \phi\left(\eta_{1}, j_{0} / q\right)$ with end points restricted to $b_{0}^{-}$. But then $\phi\left(\phi^{-1}\left(T_{j_{0} / q}, j_{0} / q\right), j_{1} / q\right)$ must contain a connected component $T_{j_{1} / q}$ satisfying $(\alpha)$ and $(\beta)$ and hence $g\left(\phi^{-1}\left(T_{j_{1} / q}, j_{1} / q\right)\right)$ has a component which is the required set (see figure $8 \mathrm{~g}$ ). The proof when $j_{1}<j_{0}$ is symmetric to the above proof.

Let $B_{1}=g^{-1}\left(T_{1}\right)$. Then by properties $(\alpha),(\beta)$ of $T_{1}$ we see that if we let $z \in A$ move around $\partial B_{1}$ and compute the total change in the angle between the vector $z-g(z)$ and the $x$-axis, the result will be $\pm 2 \pi$ (depending on orientation) (see figure 9). Hence $g$ must have a fixed point $z_{1} \in B_{1} \cap T_{1}$. Moreover, since $T_{1} \subseteq S_{1}$ we see that $\phi\left(z_{1},[0,1]\right)$ must be contractible in $A \sim\left\{\zeta_{0}, \zeta_{1}\right\}$ and since $\zeta_{0}, \zeta_{1} \notin T_{1}, z_{1} \notin\left\{\zeta_{0}, \zeta_{1}\right\}$.

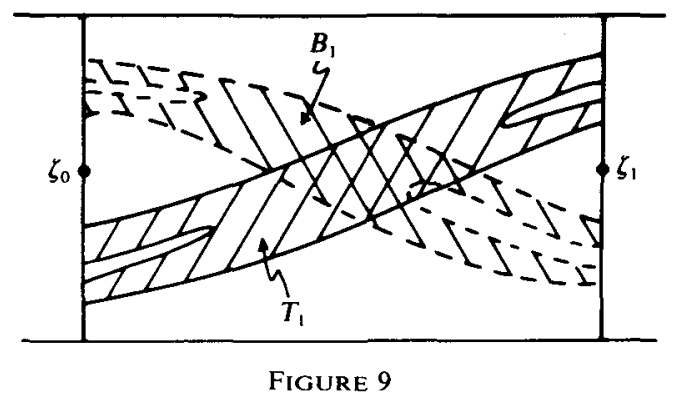

Since $z_{1}$ is a fixed point of $g$, it must be a $p / q$-periodic point of $f$. Since $\mathcal{O}\left(f, z_{0}\right)$ contains no points in $B \sim\left\{\zeta_{0}, \zeta_{1}\right\}$ we see that $z_{1} \notin \mathcal{O}\left(f, z_{0}\right)$. Finally, since $\phi\left(z_{1},[0,1]\right)$ is contractible in $A \sim\left\{\zeta_{0}, \zeta_{1}\right\}$ it follows that

$$
\pi_{1}\left(f^{i}\left(\zeta_{0}\right)\right)<\pi_{1}\left(f^{i}\left(z_{1}\right)\right)<\pi_{1}\left(f^{i}\left(\zeta_{1}\right)\right)
$$

for $i=0, \ldots, q$ and hence $z_{1}$ must be a $p / q$-Birkhoff periodic point of $f$. In case a, as noted above, we have that $\pi_{1}\left(f^{i}\left(\eta_{0}\right)\right)<\pi_{1}\left(f^{i}\left(z_{1}\right)\right)<\pi_{1}\left(f^{i}\left(\eta_{1}\right)\right)$ as well. (This will be used in case 2 below.)

To obtain the stability portion of the lemma, we note that if $\tilde{f}$ is sufficiently close to $f$ and $\tilde{g}(\cdot) \equiv \tilde{f}(\cdot)-(p, 0)$ then the index of $\tilde{g}: B_{1} \rightarrow A$ will still be non-zero and hence $\tilde{g}$ will have a fixed point $\tilde{z}_{1} \in \tilde{g}\left(B_{1}\right) \cap B_{1}$. Since $T_{1} \cap B_{1}$ is contained in the interior of $B$ (relative to $A$ ) this will also hold for $\tilde{g}\left(B_{1}\right) \cap B_{1}$ and hence

$$
\pi_{1}\left(\tilde{f}^{i}\left(\zeta_{0}\right)\right)<\pi_{1}\left(\tilde{f}^{i}\left(\tilde{z}_{1}\right)\right)<\pi_{1}\left(\tilde{f}^{i}\left(\zeta_{0}\right)\right)
$$

for $i=0, \ldots, q$ when $\tilde{f}$ is sufficiently close to $f$. It then follows as above (even though 
$\zeta_{0}, \zeta_{1}$ are not necessarily near periodic points of $\tilde{f}$ ) that $\tilde{z}_{1}$ is a $p / q$-Birkhoff periodic point of $\tilde{f}$ and the proof of case 1 is complete.

Case 2. In this case we know $\pi_{1}\left(f^{i}\left(\eta_{0}\right)\right)<\pi_{1}\left(f^{i}\left(\eta_{1}\right)\right)$ for $i=0, \ldots, q$. Hence when $\pi_{1}\left(\zeta_{0}\right) \leq \pi_{1}\left(\eta_{0}\right)<\pi_{1}\left(\zeta_{1}\right)$ we may apply lemma $3^{\prime}$ to obtain $\tilde{\phi}: A \times \mathbb{R} \rightarrow A$ associated with $g$ which has $\tilde{\phi}\left(\eta_{0}, t\right)=\eta_{0}, \tilde{\phi}\left(\zeta_{1}, t\right)=\zeta_{1}$ for all $t$. Then $\tilde{\phi}\left(\zeta_{0},[0,1]\right)$ is not contractible in $A \sim\left\{\eta_{0}\right\}$, but $\tilde{\phi}\left(\zeta_{0},[0,1]\right)$ and $\tilde{\phi}\left(\eta_{1},[0,1]\right)$ do not link. Hence we may apply case la above taking $B=\left\{z \in A: \pi_{1}\left(\eta_{0}\right) \leq \pi_{1}(z) \leq \pi_{1}\left(\zeta_{1}\right)\right\}$ and obtaining a fixed point $z_{1}$ of $g$. From case la we know that $\tilde{\phi}\left(z_{1},[0,1]\right)$ links with none of the $\tilde{\phi}$ orbits of $\zeta_{0}, \zeta_{1}, \eta_{0}$ or $\eta_{1}$. The rest of the proof proceeds as before.

The case when $\pi_{1}\left(\zeta_{0}\right)<\pi_{1}\left(\eta_{1}\right) \leq \pi_{1}\left(\zeta_{1}\right)$ is, of course symmetric to the above and the proofs of the claim and the lemma are complete.

\section{Proof of theorem 1}

Let $f: A \rightarrow A$ be a twist map, $p, q$ relatively prime integers and $w_{0} \in A$ a $p / q$-periodic point of $f$. We may assume $w_{0}$ is not a Birkhoff periodic point since if it were we would be done.

Next we note that since $f$ has a $p / q$-periodic point we have that $p / q \in$ $\left[\rho_{0}(f), \rho_{1}(f)\right]$. We may assume $p / q \in\left(\rho_{0}(f), \rho_{1}(f)\right)$ since if $\rho_{i}(f)=p / q, i=0$ or 1 then $\left.f\right|_{\{(x, i): x \in \mathbb{R}\}}$ is a lift of a circle diffeomorphism with rotation number $p / q$ and the existence of the $p / q$-Birkhoff periodic point follows from the usual arguments for circle maps (see [12]). Moreover, we may assume that for each $\alpha \in \mathbb{R}$ there exists $y_{\alpha} \in(0,1)$ such that $\pi_{1}\left(f\left(\alpha, y_{\alpha}\right)\right)=\alpha+p / q$. If this is not the case for the given map $f$ then we may extend $f$ to a map $f_{1}: A_{1} \rightarrow A_{1}$ where $A_{1}=\left\{(x, y) \in \mathbb{R}^{2}:-\frac{1}{2} \leq y \leq \frac{3}{2}\right\}, f_{1}$ is a twist map satisfying the above condition and $f$ and $f_{1}$ agree on $A$. Since $\rho_{0}(f)<p / q<\rho_{1}(f)$, if we can find a $p / q$-Birkhoff periodic point for $f_{1}$ on $A_{1}$ then in fact it must have orbit in $A$ (see figure 10).

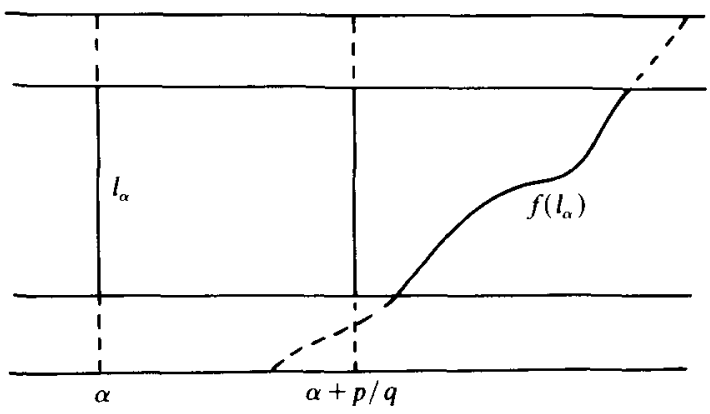

Figure 10

Claim 1. There exists a $C^{2}$ homotopy $H: A \times[0,1] \rightarrow A$ satisfying

(a) $H(\cdot, 0)=f(\cdot)$;

(b) for all $s \in[0,1], H(\cdot, s)$ is a twist map;

(c) for all $s \in[0,1], w_{0}$ is a $p / q$-periodic point of $H(\cdot, s)$;

(d) $H(\cdot, 1)$ has a $p / q$-Birkhoff periodic point. 
Proof of claim 1. Fix $z_{0} \in A$ so that $z_{0}$ is in the interior of $A$,

$$
\left\{\pi_{1}\left(z_{0}\right)+j p / q, j=0, \ldots, q-1\right\} \cap\left(\left\{\pi_{1}(w): w \in \mathscr{O}\left(f, w_{0}\right)\right\} \cup \mathbb{Z}\right)=\varnothing
$$

and $\pi_{1}\left(f\left(z_{0}\right)\right)=\pi_{1}\left(z_{0}\right)+p / q$. Fix $y_{i} \in[0,1]$ so that $\pi_{1}\left(f\left(\pi_{1}\left(z_{0}\right)+i p / q, y_{i}\right)\right)=$ $\pi_{1}\left(z_{0}\right)+(i+1) p / q$ for $i=0, \ldots, q-1$ (so $y_{0}=\pi_{2}\left(z_{0}\right)$ ). For any $\varepsilon>0$ we may define $h_{i, s}: A \rightarrow A$, one parameter families of diffeomorphisms, $i=1, \ldots, q, s \in[0,1]$ so that

(1) for all $s \in[0,1], i=1, \ldots, q, h_{i, s}(x+1, y)=h_{i, s}(x, y)+(1,0)$ for all $(x, y) \in A$;

(2) for all $s \in[0,1], i=1, \ldots, q$, $\operatorname{support}\left(h_{i, s}-\right.$ identity) $\cap\{(x, y) \in A: 0 \leq x<1\}$ is contained in $\left\{(x, y) \in A:\left|x-\left(\pi_{1}\left(z_{0}\right)+i p / q-\left[\pi_{1}\left(z_{0}\right)+i p / q\right]\right)\right|<\varepsilon\right\} \quad$ (where $[\cdot]$ denotes the greatest integer function) and $h_{i,}(\cdot)$ is $C^{\infty}$ on $A \times[0,1]$,

(3) for all $s \in[0,1], i=1, \ldots, q$, and all $(x, y) \in A, \pi_{1}\left(h_{i, s}(x, y)\right)=x$,

(4) $i=1, \ldots, q, h_{i, 0}=$ identity and

$$
\begin{aligned}
& \pi_{2}\left(h_{i, 1}\left(\pi_{1}\left(z_{0}\right)+i p / q-\left[\pi_{1}\left(z_{0}\right)+i p / q\right], \pi_{2}\left(f\left(\pi_{1}\left(z_{0}\right)+(i-1) p / q, y_{i-1}\right)\right)\right)\right. \\
& \quad= \begin{cases}y_{i} & \text { if } i=1, \ldots, q-1, \\
y_{0} & \text { if } i=q .\end{cases}
\end{aligned}
$$

Choose $\varepsilon$ so small that

$$
\left(\bigcup_{i=1}^{q} \bigcup_{s \in[0,1]} \operatorname{support}\left(h_{i, s}-\text { identity }\right)\right) \cap\left(\mathcal{O}\left(f, w_{0}\right) \cup\{(j, y) \in A: j \in \mathbb{Z}\}\right)=\varnothing
$$

and define

$$
\begin{gathered}
H: A \times[0,1] \rightarrow A \\
H(z, s)=h_{q, s} \circ f \circ h_{q-1, s} \circ h_{q-2, s} \circ \cdots \circ h_{1, s}(z) .
\end{gathered}
$$

Then $H$ has properties (a)-(d) above. In particular, for all $s \in[0,1], w_{0}$ is a $p / q$ periodic point of $H(\cdot, s)$ since $f$ and $H(\cdot, s)$ agree on a neighbourhood of $\mathcal{O}\left(f, w_{0}\right)$ and $H(\cdot, s)$ is a twist map since the composition of a twist map with a map preserving the $x=$ constant foliation is a twist map. The map $H(\cdot, 1)$ has $z_{0}$ as a $p / q$-periodic point by condition (4) above and $z_{0}$ is a $p / q$-Birkhoff periodic point since any $\zeta \in \mathcal{O}\left(H(\cdot, 1), z_{0}\right)$ satisfies $\pi_{1}(H(\zeta, 1))=\pi_{1}(\zeta)+p / q$ again by condition (4). Hence, $H$ is the required homotopy and the proof of the claim is complete.

Let $\Xi=\left\{s \in[0,1]\right.$ : for all $s_{1} \geq s, H\left(\cdot, s_{1}\right)$ has a $p / q$-Birkhoff periodic point $\}$. Since $1 \in \Xi \neq \varnothing$, if we can show that the point $s_{1}=\inf \{s \in[0,1]: s \in \Xi\}$ is in the interior of $\Xi$ i.e. that $\Xi$ is open and closed, then we must have $\Xi=[0,1]$. Hence $H(\cdot, 0)=f(\cdot)$ would have a $p / q$-Birkhoff periodic point and the proof would be complete.

Claim 2. $\Xi$ is closed.

Proof of claim 2. This follows immediately from lemma 1.

Claim 3. $\Xi=[0,1]$.

Proof of claim 3. Suppose $\Xi \neq[0,1]$. Then $s_{1}=\inf \{s \in[0,1]: s \in \Xi\}>0$. By claim 2 we have that $s_{1} \in \Xi$ and hence that $H\left(\cdot, s_{1}\right)$ has a $p / q$-Birkhoff periodic point $\zeta_{0} \in A$. By construction, $w_{0}$ is a $p / q$-periodic point of $H\left(\cdot, s_{1}\right)$ which is not a Birkhoff periodic point. Hence, we may apply lemma 4 to show that $H\left(\cdot, s_{1}\right)$ has another $p / q$-Birkhoff periodic point $z_{1} \in A$ with $z_{1} \notin \mathcal{O}\left(H\left(\cdot, s_{1}\right), z_{1}\right)$ and, more importantly, for $s$ sufficiently close to $s_{1}$, the stability statement in lemma 4 implies $H(\cdot, s)$ also 
has a $p / q$-Birkhoff periodic point. Hence $s_{1}$ is in the interior of $\Xi$ contradicting the definition of $s_{1}$. This contradiction implies that we must have $s_{1}=0$, i.e. $\Xi=[0,1]$, and the proof of the claim is complete.

As noted above, $0 \in \Xi$ implies that $H(\cdot, 0)=f(\cdot)$ has a $p / q$-Birkhoff periodic point and the proof of theorem 1 is complete.

\section{Some related theorems}

In this section we show

TheOREM 4. Suppose $f: A \rightarrow A$ is a twist map and $p, q$ are relatively prime integers. If every $r / s$-periodic point of $f$ satisfies $s=q$ or $s>3 q / 2$ then every $p / q$-periodic point of $f$ is a $p / q$-Birkhoff periodic point.

Remark. The theorem states that a periodic orbit of a twist map, with period significantly smaller than all larger periods, will be a Birkhoff periodic orbit. Since the possible periods are always contained between the rotation numbers of the map restricted to the boundaries, we may give conditions under which the hypotheses hold as follows:

Let $\mathscr{F}_{n}$ denote the Farey series of order $n$, i.e. $\mathscr{F}_{n}$ is a series of irreducible fractions between zero and one in ascending order with denominator less than or equal to n. So

(see [11]).

$$
\mathscr{F}_{1}=\left\{\frac{0}{1}, \frac{1}{1}\right\}, \quad \mathscr{F}_{2}=\left\{\frac{0}{1}, \frac{1}{2}, \frac{1}{1}\right\}, \quad \mathscr{F}_{3}=\left\{\frac{0}{1}, \frac{1}{3}, \frac{1}{2}, \frac{2}{3}, \frac{1}{1}\right\}, \cdots .
$$

Corollary. Suppose $f: A \rightarrow A$ is a twist map, $p, q$ are relatively prime integers and $\rho_{0}(f), p / q, \rho_{1}(f)$ are consecutive elements in $\mathscr{F}_{n}$ for some $n$. If $\rho_{0}(f)=r_{0} / s_{0}, \rho_{1}(f)=$ $r_{1} / s_{1}$ in lowest form and $s_{0}>3 q / 2, s_{1}>3 q / 2$ then all $p / q$-periodic points of $f$ (if any) are $p / q$-Birkhoff periodic points.

Proof of the corollary. Suppose $r / s \in \mathscr{F}_{n+i} \sim \mathscr{F}_{n+i-1}$ for $i>0$ and $r_{0} / s_{0}<r / s<p / q$. Then, by theorems 29-31 of [11], $r / s=\left(a_{1}+a_{2}\right) /\left(b_{1}+b_{2}\right)$ where $a_{1} / b_{1}, a_{2} / b_{2}$ are consecutive elements of $\mathscr{F}_{n+i-1}$ and $a_{1}+a_{2}, b_{1}+b_{2}$ are relatively prime. But then $a_{1} / b_{1}, a_{2} / b_{2} \in\left[r_{0} / s_{0}, p / q\right]$ and hence $a_{1} / b_{1} \in \mathscr{F}_{n}$ iff $a_{1} / b_{1}=r_{0} / s_{0}$ and $a_{2} / b_{2} \in \mathscr{F}_{n}$ iff $a_{2} / b_{2}=p / q$. Hence $b_{1} \geq q$ and $b_{2} \geq q$ so $s \geq 2 q$. Similarly, if $p / q<r / s<r_{1} / s_{1}$ then $s \geq 2 q$ so $f, p, q$ satisfy the hypotheses of theorem 4 and the proof of the corollary is complete.

Remark. Essentially the theorem and corollary above say that if $f: A \rightarrow A$ is a twist map and $f$ is not 'twisting' very much, then the periodic orbits with smallest period must be Birkhoff periodic orbits. We know of no examples implying that the conditions of theorem 4 are the best possible, however if $f$ is area-preserving then the theorem can be improved significantly.

THEOREM 5. If $f: A \rightarrow A$ is an area-preserving twist map, $p, q$ are relatively prime integers and if every $r / s$-periodic point of $f$ has $s \geq q$ then every $p / q$-periodic point of $f$ is a $p / q$-Birkhoff periodic point. 
The proof of theorem 4 follows from ideas similar, but much easier technically, to those in theorem 1 . The proof of theorem 5 is vaguely related to the 'graph-theoretical' approach to Šarkovskiǐ's theorem in [6].

Proof of theorem 4. Fix $f: A \rightarrow A$ and $p, q$ as in the theorem. Suppose $z_{0} \in A$ is a $p / q$-periodic point, but $z_{0}$ is not a $p / q$-Birkhoff periodic point of $f$. Then we fix $\zeta_{1}, \zeta_{2} \in \mathcal{O}\left(f, z_{0}\right)$ so that $\pi_{1}\left(\zeta_{1}\right)<\pi_{1}\left(\zeta_{2}\right), \pi_{1}\left(f\left(\zeta_{1}\right)\right)>\pi_{1}\left(f\left(\zeta_{2}\right)\right)$ and $\pi_{1}\left(f^{j}\left(\zeta_{1}\right)\right)<$ $\pi_{1}\left(f^{j}\left(\zeta_{2}\right)\right)$ for some $j, 1<j \leq q / 2+1$. Then there exists an integer $r$ so that $f^{k}\left(\zeta_{2}\right)=$ $\zeta_{1}+(r, 0)$ for some $k, j \leq k \leq 3 q / 2, k \neq q$.

Now fix $N>0$ so large that $\pi_{1}\left(f^{i}\left(\pi_{1}\left(\zeta_{2}\right), 1\right)\right)<\pi_{1}\left(f^{i}\left(\pi_{1}\left(\zeta_{1}\right)+N, 0\right)\right)$ for $i=$ $0, \ldots, 2 q$ and let $B=\left\{(x, y): \pi_{1}\left(\zeta_{1}\right) \leq x \leq \pi_{1}\left(\zeta_{1}\right)+N\right\}$. Let $g: A \rightarrow A$ be defined for all $z \in A$ by, $g(z)=f^{k}(z)-(r, 0)$. Then $g\left(\zeta_{2}\right)=\zeta_{1}$. Let

$$
J_{1}=\left\{\left(\pi_{1}\left(\zeta_{1}\right), y\right): \text { for all } y_{1} \geq y, g\left(\pi_{1}\left(\zeta_{1}\right), y_{1}\right) \in B\right\}
$$

and

$$
J_{2}=\left\{\left(\pi_{1}\left(\zeta_{1}\right)+N, y\right): \text { for all } y_{1} \leq y, g\left(\pi_{1}\left(\zeta_{1}\right)+N, y_{1}\right) \in B\right\}
$$

Then the component $T$ of $g(B) \cap B$ with boundary containing $g\left(J_{1}\right) \cup g\left(J_{2}\right)$ must have a fixed point. This is easily seen by computing the change of the argument of the vector $z-g(z)$ as $z$ moves around the boundary of $g^{-1}(T)$, this change is $\pm 2 \pi$ depending on orientation (see figure 11).

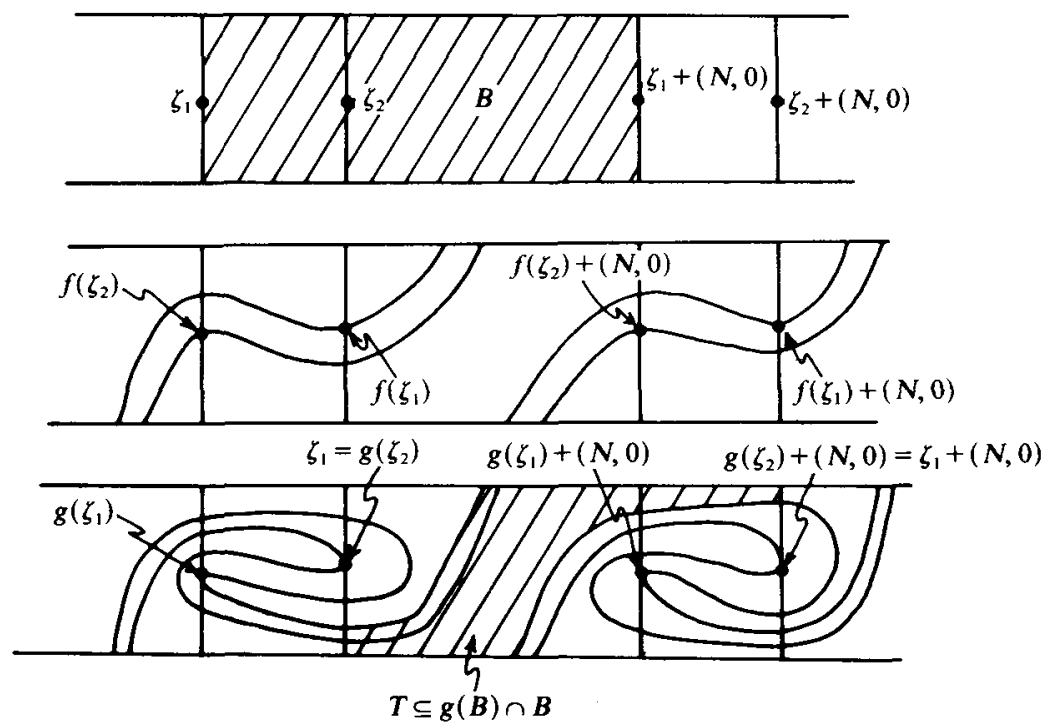

Figure 11

But this fixed point of $g$ corresponds to an $r / k$-periodic point of $f$. Hence, if $f$ has no $r / s_{1}$-periodic points with $s_{1} \neq q, s_{1} \leq 3 q / 2$ then every $p / q$-periodic point of $f$ must be a $p / q$-Birkhoff periodic point and the proof is complete.

Proof of theorem 5. Fix $f: A \rightarrow A$ an area preserving twist map, $p, q$ relatively prime integers and $z_{0}$ a $p / q$-periodic point of $f$. Let

$$
\left\{\zeta_{0}, \ldots, \zeta_{q-1}\right\}=\mathcal{O}\left(f, z_{0}\right) \cap\left\{z \in A: \pi_{1}(z) \in[0,1)\right\}
$$


and number so that $\pi_{1}\left(\zeta_{0}\right) \leq \pi_{1}\left(\zeta_{1}\right) \leq \cdots \leq \pi_{1}\left(\zeta_{q-1}\right)$. We may assume, by changing variables slightly if necessary, that $\pi_{1}\left(\zeta_{i}\right) \neq \pi_{1}\left(\zeta_{i+1}\right)$ for $i=0, \ldots, q-1$. We define a directed graph with $\zeta_{0}, \ldots, \zeta_{q-1}$ as nodes as follows:

There is an edge from $\zeta_{i}$ to $\zeta_{j}$ if and only if

(a) $\pi_{1}\left(f\left(\pi_{1}\left(\zeta_{i}\right), 0\right) \in\left(\pi_{1}\left(\zeta_{j-1}\right)+s, \pi_{1}\left(\zeta_{j}\right)+s\right]\right.$ for some $s \in \mathbb{Z}$; or

(b) $\pi_{1}\left(f\left(\pi_{1}\left(\zeta_{i}\right), 1\right) \in\left[\pi_{1}\left(\zeta_{j}\right)+s, \pi_{1}\left(\zeta_{j+1}\right)+s\right)\right.$ for some $s \in \mathbb{Z}$.

Hence each $\zeta_{i}$ has at least one edge exiting it, moreover if $z_{0}$ is not a $p / q$-Birkhoff periodic point then some $\zeta_{i}$ has two edges exiting from it since for some $\zeta_{i}, \zeta_{j}, \pi_{1}\left(\zeta_{i}\right)<$ $\pi_{1}\left(\zeta_{j}\right)$ but $\pi_{1}\left(f\left(\zeta_{i}\right)\right)>\pi_{1}\left(f\left(\zeta_{j}\right)\right)$ (see figure 12 ), so

$$
\pi_{1}\left(f\left(\pi_{1}\left(\zeta_{i}\right), 0\right)\right)<\pi_{1}\left(f\left(\zeta_{j}\right)\right)<\pi_{1}\left(f\left(\zeta_{i}\right)\right)<\pi_{1}\left(f\left(\pi_{1}\left(\zeta_{i}\right), 1\right)\right)
$$

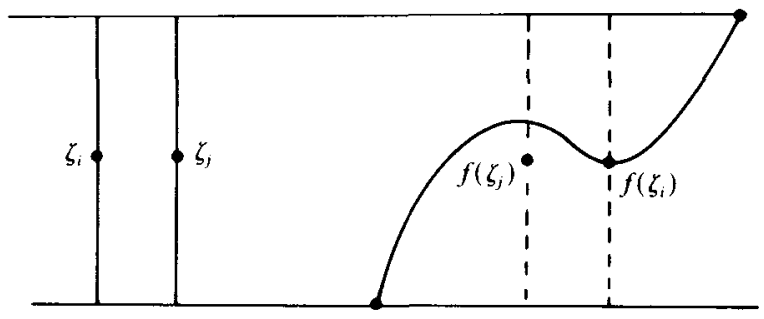

FIGURE 12

In this case the graph formed has loops of length less than $q$. But this implies that there exist rationals $r / s \in\left[\rho_{0}(f), \rho_{1}(f)\right]$ with $s<q$. By Birkhoff's theorem (theorem 2) (as noted by Birkhoff, $f: A \rightarrow A$ need only preserve a measure with non-zero density for theorem 2 to hold, see [4], so the change of variables above is no problem) we see that $f$ must have an $r / s$-periodic point. Hence, if $f: A \rightarrow A$ has no $r / s$-periodic points with $s<q$ then every $p / q$-periodic point of $f$ is a $p / q$-Birkhoff periodic point.

Acknowledgements. The author would like to thank all of those who listened patiently and offered suggestions and encouragement during this work. Particular thanks to C. Conley, E. Mansfield and D. Terman. A special thanks to M. Handel for suggestions simplifying and extending lemma 4 which removed many technical difficulties and for suggesting the possibility of theorems of $\S 6$. Finally, thanks to David Bernstein for pointing out an error in lemma 3'.

The author was sponsored by the United States Army under contract no. DAAG2980-C-0041, and a National Science Foundation Postdoctoral Fellowship.

\section{REFERENCES}

[1] D. Asimov \& J. Franks. Unremovable closed orbits. Preprint.

[2] S. Aubry. Theory of the devil's staircase. Seminar on the Riemann Problem and Complete Integrability, 1978/79, (Ed. D. O. Chudrovsky), Lecture Notes in Math. 925 Springer: Berlin-Heidelberg-New York.

[3] D. Bernstein. Birkhoff periodic orbits for twist maps with graph intersection property. Ergod. Th. \& Dynam. Sys. To appear. 
[4] G. P. Birkhoff. Proof of Poincaré's geometric theorem. In George David Birkhoff: Collected Mathematical Papers, Vol. 1. Dover Publishing Inc: New York, 1968, p. 673.

[5] G. D. Birkhoff. An extension of Poincaré's last geometric theorem. In George David Birkhoff: Collected Mathematical Papers, Vol. 2. Dover Publishing Inc: New York, 1968, p. 252.

[6] L. Block, J. Guckenheimer, M. Misureiwicz \& L-S. Young. Periodic points and topological entropy of one dimensional maps. Preprint.

[7] M. Brown \& W. D. Neumann. Proof of the Poincaré-Birkhoff fixed point theorem. Michigan Math. J. 24 (1977), 21-31.

[8] P. Carter. An improvement of the Poincaré-Birkhoff fixed point theorem. Trans. Amer. Math. Soc. 269 no. 1, (1982), 285-299.

[9] A. Chenciner. Sur un enoncé dissipatif du théoremè geometric de Poincaré-Birkhoff. C. R. Acad. Sc. Paris, 294 Sec. 1, (1982), 243-245.

[10] A. Chenciner. Bifurcations de points fixes elliptiques. Preprint.

[11] G. H. Hardy \& E. M. Wright. An Introduction to the Theory of Numbers, (fifth edition). Oxford University Press: Oxford, 1979.

[12] M. Herman. Sur la conjugaison differentiable des diffeomorphisms du cercle a des rotations. Publ. Math. I.H.E.S. 491979.

[13] M. Herman. Introduction a l'etude des courbes invariantes par les diffeomorphisms de l'anneau, Vol. 1. Preprint.

[14] A. Katok. Some remarks on Birkhoff and Mather twist map theorems. Ergod. Th. \& Dynam. Sys. 2 (1982), 185-192.

[15] J. N. Mather. Existence of quasi-periodic orbits for twist homomorphisms. Topology (1982).

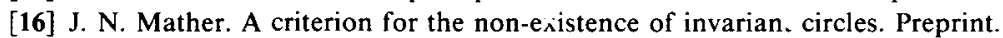

[17] T. Matsuoka. The number and linking of periodic solutions of periodic systems. Invent. Math. 70 (1983), 319-340. 